\title{
RECENT RESULTS ON POLARIZATION OF T TAURI STARS AND OTHER YOUNG STELLAR OBJECTS
}

\author{
PIERRE BASTIEN and FRANÇOIS MÉNARD \\ Observatoire du Mont Mégantic, and Département de Physique, \\ Université de Montréal \\ B. P. 6128, Succ. A, Montréal, Québec, H3C 3J7 \\ Canada
}

\begin{abstract}
Recent results on observations and models of polarization of $\mathrm{T}$ Tauri stars (TTS) and other young stellar objects (YSO's) are presented. In particular, the difference in polarization properties between the classical T Tauri stars (CTTS) and the weak-line T Tauri stars (WTTS) is made. A correlation between polarization and rotation period is searched for but not found. Observations of polarization maps for many young stars are considered. Two types of models have been proposed to explain these observations. Current evidence favors single and multiple scattering in flattened, optically thick, structures, i.e. disks, around many TTS. In particular, the size of the optically thick part of the disks, and their inclination to our line of sight can determined for published polarization maps.
\end{abstract}

\section{Introduction}

The observations for the first important paper on linear polarization observations of TTS were carried out here, at the Byurakan Observatory, more than 25 years ago (Vardanian 1964). The observations of RY Tau are plotted in Fig. 1 as a function of time. The star shows strong variations in both $\mathrm{P}$ (from $2 \%$ to $4.5 \%$ ) and $\theta$ (from $0^{\circ}$ to $80^{\circ}$ ). The main conclusions of the paper are best summarized by this sentence from the English abstract to the paper: "The observed variations of the polarization parameters of T and RY Tau as well as the value of the ratio P/Av for RY Tau permit to suggest that these stars have intrinsic polarization". This suggestion has been confirmed by subsequent observations for these two and many other TTS.

A discussion of subsequent polarization observations of TTS with a detailed review of their polarization properties and models has been given recently by Bastien (1988). In this paper, we point out a few new results on this subject. General review papers on TTS have been written by Bertout (1989) and by Appenzeller and Mundt (1990).

\section{New Results}

X-ray surveys of dark clouds have led recently to the discovery of another group of low mass TTS, the weak-line T Tauri stars (WTTS)(Walter et al. 1988). WTTS are believed to 


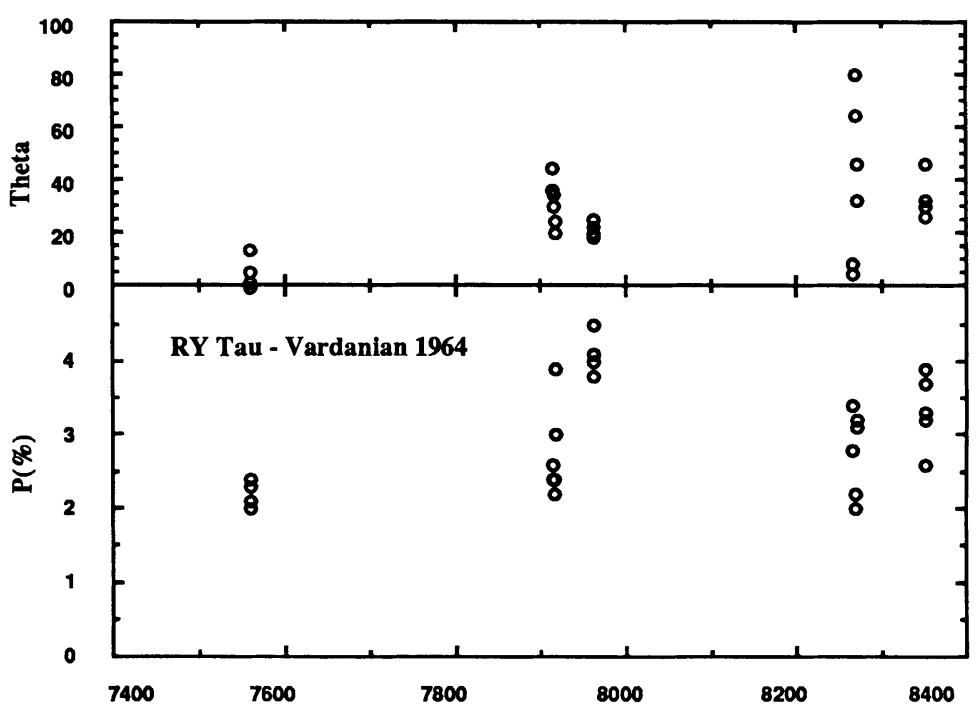

J.D.

Figure 1. Linear polarization of RY Tau measured by Vardanian (1964) The three observing seasons, 1961, 1962 and 1963 (August to November) can be found easily. The error bars are about $0.3 \%$. The data shows clearly the polarization variability of the star.

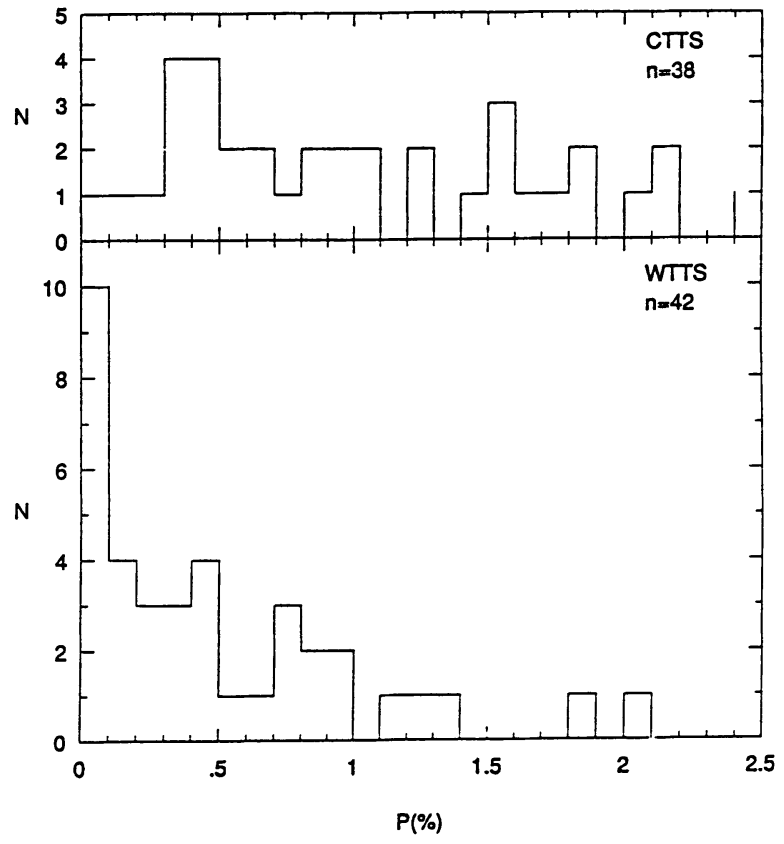

Figure 2. Histograms of linear polarization for CTTS and WTTS in the Taurus-Auriga dark cloud complex. 
have no or very weak circumstellar disks, which distinguishes them from the classical TTS (CTTS) whose main properties are believed to be due to the presence of a disk. Infrared observations provide support for this interpretation, as the WTTS appear to have much weaker infrared excesses (Strom et al. 1989). Another check is to measure and compare the linear polarization of the two groups of stars since the polarization is due to circumstellar dust. In Fig. 2, we present two histograms of polarization data for the CTTS and WTTS in the Taurus-Auriga T associations. CTTS have larger values of polarization which are clearly different from zero, whereas the WTTS show a strong peak at zero polarization. This implies that most (but not all) WTTS have no or very little circumstellar material.

The results of polarization surveys of TTS (Bastien 1982, 1985) have been used to search for correlations between polarization and other properties of these stars. In particular, there is a very strong correlation between polarization and the infrared excess. Since then, rotation periods have been determined for many TTS (see Herbst 1990 and references therein). We present in Fig. 3 a plot of polarization against rotation period. As can be seen, there seems to be no correlation.

Linear polarization maps of nearly 60 YSO's have been published in the past few years. In these maps, the polarization vectors are usually perpendicular to the radius vector from the central illuminating source. Such a centrosymmetric pattern is typical of reflection nebulae where single scattering is responsible for the polarization since the polarization vectors are usually perpendicular to the scattering plane. However, about $60 \%$ of these maps show a pattern of aligned polarization vectors close to the central source (Bastien and Ménard 1988, 1990, hereafter BM1 and BM2 respectively). The polarization reaches typically $10 \%-15 \%$ in this region, the highest value noted so far being $30 \%$.

Two interpretations have been proposed for the patterns of aligned polarization vectors. (1) Dichroic extinction by aligned nonspherical grains in a circumstellar disk has been suggested by many authors (e. g. Warren-Smith 1987). Various alignment mechanisms are possible. Alignment by a toroidal magnetic field is usually preferred. However, no model calculations for this interpretation have been performed. (2) Multiple scattering in and around an optically thick circumstellar disk was proposed by BM1, where they presented calculations done under the assumption that only two scatterings occur in the disk, and showed that it is possible to obtain patterns of aligned vectors with multiple scattering, provided one has an optically thick surface.

There are many objections which can be raised against dichroic extinction (BM1, BM2). No polarization observations support the presence of aligned grains around TTS and YSO's. This means that magnetic fields, if present, are not efficient at aligning grains in the circumstellar environment. However, all of the arguments against dichroic extinction support the idea of scattering on dust grains. In particular, circular polarization has been predicted and detected in the region (disk) where multiple scattering is occurring (Ménard, Bastien, and Robert 1988). When the observed maps are compared with computed maps, one can obtain the inclination of the disk and also the size of the disk for which the optical depth is $\geq 1$ (BM2). The BM1 model has now been confirmed with a Monte Carlo code which includes as many scatterings as there should be for given geometry and dust properties (Ménard 1989, Ménard and Bastien 1990). In Fig. 4 we show a computed map which corresponds to an average inclination angle of $93.2^{\circ}$. The density law used decreases linearly with distance $r$ from the star in the equatorial plane, and exponentially with distance $z$ from that plane. Graphite grains with a radius of $0.2 \mu \mathrm{m}$ have been used, giving an optical depth in the plane of the disk of 36.

We are grateful to the Natural Sciences and Engineering Research Council (NSERC) of Canada for financial assistance. 


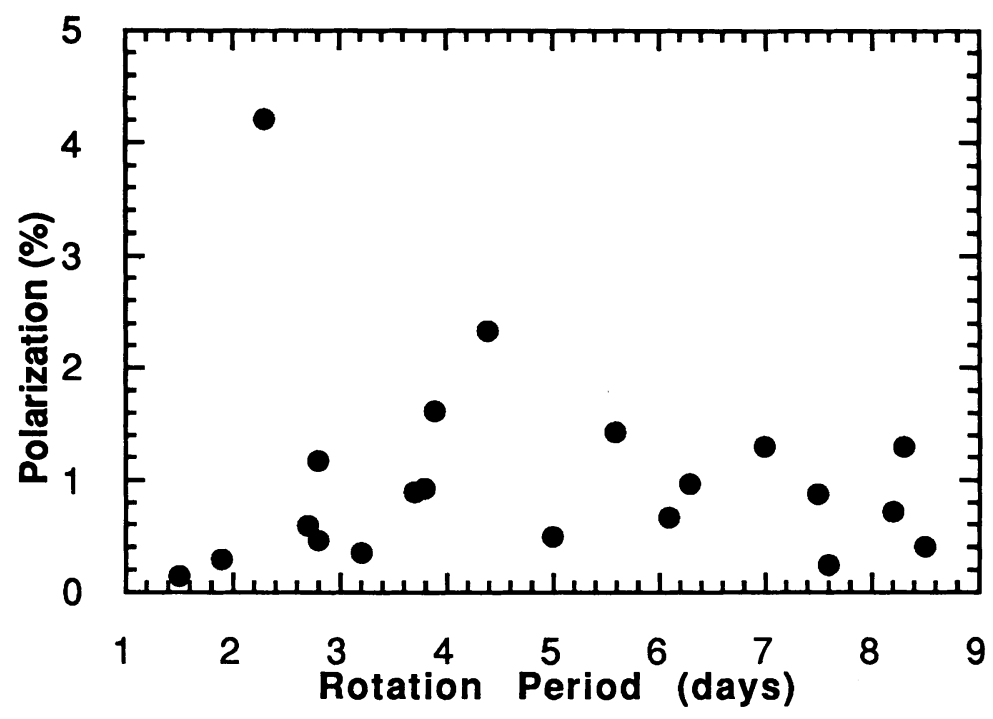

Figure 3. Polarization in a red passband of TTS plotted against their rotation periods. The star with a large polarization and a small rotation period is $\mathrm{LH} \alpha 332-20$.

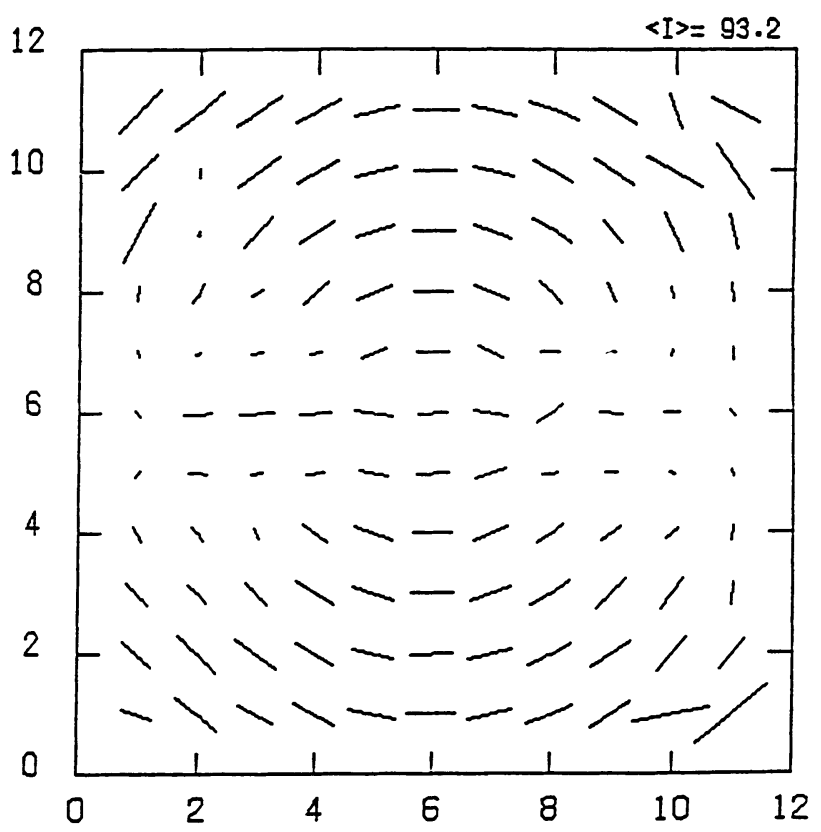

Figure 4. Polarization map computed with the Monte Carlo code for an optically thick disk with a $r^{1}$ density law and an exponential decrease with distance $z$ from the equatorial plane. The region shown corresponds to $10 \mathrm{AU}$ on the side. 


\section{REFERENCES}

Appenzeller, I., and Mundt, R. (1990) 'Observational Properties of T Tauri Stars', Astron. Astrophys. Rev., in press.

Bastien, P. (1982) 'A Linear Polarization Survey of T Tauri Stars', Astron. Astrophys. Suppl., 48, 153-164, and 48, 513-518.

Bastien, P. (1985) 'A Linear Polarization Survey of Southern T Tauri Stars', Astrophys. J. Suppl., 59, 277-291.

Bastien, P. (1988) 'Polarization Properties of T Tauri Stars and Other Pre-Main Sequence Objects', in G. V. Coyne et al. (eds.), Polarized Radiation of Circumstellar Origin, Vatican Press, Vatican City, 541-582.

Bastien, P., and Ménard, F. (1988) 'On the Interpretation of Polarization Maps of Young Stellar Objects', Astrophys. J., 326, 334-338 (BM1).

Bastien, P., and Ménard, F. (1990) 'Parameters of Disks Around Young Stellar Objects From Polarization Observations', submitted (BM2).

Bertout, C. (1989) 'T Tauri Stars: Wild as Dust', Ann. Rev. Astron. Astrophys., 27, 351-395.

Herbst, W. (1990) 'The Variability of T Tauri Stars', this volume.

Ménard, F. (1989) 'Étude de la polarisation causée par des grains dans les enveloppes circumstellaires denses', Ph. D. Thesis, Univ. de Montréal.

Ménard, F., and Bastien, P. (1990), in preparation.

Ménard, F., Bastien, P., and Robert, C. (1988) 'Detection of Circular Polarization in R Monocerotis and NGC 2261: Implications for the Polarization Mechanism.', Astrophys. J., 335, 290-294.

Strom, K. M., Strom, S. E., Edwards, S., Cabrit, S., Strutskie, M. F. (1989) 'Circumstellar Material Associated With Solar-type Pre-Main-Sequence Stars: A Possible Constraint on the Time Scale for Planet Building', Astron. J., 97, 14511470.

Vardanian, R. A. (1964) 'The Polarization of T and RY Tau', Contrib. Byurakan Obs., 35, 3-23 (in Russian).

Walter, F. M., Brown, A., Mathieu, R. D., Myers, P. C., Vrba, F. J. (1988) 'X-Ray Sources in Regions of Star Formation. III. Naked T Tauri Stars Associated With the Taurus-Auriga Complex', Astron. J., 96, 297-325.

Warren-Smith, R. F. (1987) 'Magnetic Fields and the Optical Polarization of Star Formation Regions', Quart. J. R. A. S., 28, 298. 
LADA: Can you apply your modelling technique to $T$ Tauri stars without such extended reflection nebulae to obtain disk inclinations? Such observations would be very important for understanding the energy budget of individual YSO's. Certainly with knowledge of disk inclination we would be able to obtain more accurate estimates of the bolometric luminosities of $\mathrm{T}$ Tauri stars and perhaps estimates of their accretion luminosity as well.

BASTIEN: Yes, provided one has high spatial resolution observations of these stars. For example, if polarization measurements can be made with the Space Telescope one should be able to derive inclination angles for these stars which have less extended nebulosities around them.

LANG: IRAS infrared observations show evidence for circumstellar disks, and in one case (beta Pictoris) the disk has been imaged optically. Is there linear polarization for these objects and does it confirm your theory of scattering from a circumstellar disk?

BASTIEN: Yes, the linear polarization of the disk associated with beta Pictoris has been measured. The polarization is perpendicular to the disk, which means that the optical depth is so small that single scattering only is taking place in the disk.

LANG: Are there similar infrared observations of $T$ Tauri stars that show strong linear polarization for a circumstellar disk?

BASTIEN: A few young stars have been imaged in the infrared from the ground and do show evidence for a circumstellar disk. Some polarization measurements have also been made at 2 micron and show evidence for circumstellar disks.

VARDANIAN: Was the role of interstellar polarization taken into account during the modelling?

BASTIEN: No, it was not taken into account because it does not have to. The interstellar polarization is usually small (about $0.1 \%$ in Taurus to $<2 \%$ in Cygnus, for example) whereas the polarization in the models is large $(>10 \%)$ and would dominate interstellar polarization.

MITSKEVICH: Did you try to find any correlation between the polarization of $T$ Tauri stars and displacement of their molecular lines, for example CO-lines?

BASTIEN: No, I did not. 